\title{
Tactile interactions lead to coherent motion and enhanced chemotaxis of migrating cells
}

\author{
Luke Coburn $^{1,5}$, Luca Cerone ${ }^{2}$, Colin Torney ${ }^{3}$, Iain D Couzin ${ }^{3}$ \\ and Zoltan Neufeld ${ }^{4}$ \\ ${ }^{1}$ School of Mathematical Sciences and Complex and Adaptive Systems Laboratory, \\ University College Dublin, Dublin, Ireland \\ ${ }^{2}$ European Bioinformatics Institute (EMBL-EBI), Wellcome Trust Genome Campus, \\ Cambridge CB10 1SD, UK \\ ${ }^{3}$ Department of Ecology and Evolutionary Biology, Princeton University, Princeton, NJ 08544, USA \\ ${ }^{4}$ School of Mathematics and Physics, University of Queensland, Brisbane, Australia \\ E-mail: lukecoburn@gmail.com
}

\begin{abstract}
When motile cells come into contact with one another their motion is often considerably altered. In a process termed contact inhibition of locomotion (CIL) cells reshape and redirect their movement as a result of cell-cell contact. Here we describe a mathematical model that demonstrates that CIL alone is sufficient to produce coherent, collective cell migration. Our model illustrates a possible mechanism behind collective cell migration that is observed, for example, in neural crest cells during development, and in metastasizing cancer cells. We analyse the effects of varying cell density and shape on the alignment patterns produced and the transition to coherent motion. Finally, we demonstrate that this process may have important functional consequences by enhancing the accuracy and robustness of the chemotactic response, and factors such as cell shape and cell density are more significant determinants of migration accuracy than the individual capacity to detect environmental gradients.
\end{abstract}

\section{Introduction}

Collective migration of cells plays an important role in embryological development, tissue repair and cancer invasion [1-4]. Some migrating cells move as tightly connected clusters or sheets, while others form loosely associated streams in which cells make occasional temporary contacts. One classical example of such collective migration is produced by a group of highly mobile undifferentiated cells called neural crest cells that migrate through the developing embryo to distant locations and subsequently give rise to many different types of tissues [5, 6]. Multicellular organization is also common in tissue invasion by malignant tumour cells [7]. Targeting such

\footnotetext{
${ }^{5}$ Author to whom any correspondence should be addressed.
}

collective behaviour may be effective in controlling the spread of cancer [8]. Despite these widespread implications, the mechanisms by which large groups of cells can self-organize from local cell-cell interactions remains poorly understood.

Collective migration has been studied extensively in the context of animal groups, such as flocking birds, insect swarms and fish schools. Theoretical models and computer simulations of animal migration have shown that the general mechanisms responsible for the emergent group level migratory properties can be captured by simple interaction rules such as attraction, repulsion and local alignment [9-13]. One of the key differences between these migrations and collective cell migration is that cells are restricted to highly localized information about their neighbours. As larger organisms, such as birds and fish, use sight to detect and 
interact with neighbours at a distance, simulations of their motion often consider these animals as point particles that interact via non-local interaction rules. However, the only information available to cells about the location and behaviour of their neighbours comes either in the form of diffused chemical signals, that in most cases cannot provide accurate directional information, or from direct physical contact with other cells. Therefore to model realistic cell-cell interactions it is necessary to take into account the spatial extension and dynamically changing shape of the cells that cannot be described by simple point particle models.

The cytoskeleton of cells is an elaborate network of biopolymers which can readily reorient itself into a new shape. Cells move via extensions of their cytoskeletons called protrusions which attach to the substrate while contractile forces within the cell cytoskeleton drag it forward in the direction of the protrusions [14]. These protrusions also sense the environment immediately surrounding the cell. Cellular protrusions are unstable, forming and collapsing constantly in every direction. After formation a protrusion may either collapse or branch to produce further protrusions [15, 16]. Cells in motion are polarized with the protrusions on one end more stable than the other and as such the time-averaged distribution of protrusions has a generally asymmetric shape. The stability and distribution of protrusions around the cell body may be influenced by external chemical signals producing directional movement, called chemotaxis [16, 17].

The movement of cells is affected by interactions with other cells. In the case of dense confluent cell sheets such interactions primarily involve mechanical stresses due to adhesion, volume exclusion and proliferation [18-21] that can described for example by the so-called cellular Potts model (CPM) [22]. The CPM has been extended recently to represent active cell motility [23, 24]. Kabla has shown recently [24] that a model in which the mechanical interactions of the CPM are complemented with motile forces generated by polarized cells can reproduce a variety of collective sheet migration pattern observed in experiment. In loosely associated stream-like migration cell protrusions transmit biochemical signals. For neural crest cells, whose migration shares many characteristics with metastatic cancer cells, such interactions provide directional guidance that leads to coordinated migration even in the absence of external chemoattractant [6, 25-27]. When two cells touch a chemical exchange occurs and in response to this signalling, the protrusions at the site of contact collapse. As the protrusions are responsible for the motion, this causes the cells to redirect and after some time the cell reforms the lost protrusions in a new position on the cell surface while the cytoskeleton reorients itself. This type of contact inhibition of locomotion (CIL) was first observed by Abercrombie in tissue cultures [28] and loss of CIL has been proposed to contribute to the invasiveness of malignant cells [29]. Recent in vivo experiments clearly demonstrate that CIL plays a key role in the directional migration of neural crest cells [26, 30, 31]. Furthermore, in the presence of an external chemoattractant CIL was shown to promote collective chemotaxis of neural crest cells. While separated cells are unable to move in the direction of gradient without interacting with other cells, the efficiency of collective migration of a cell population towards the chemoattractant was found to be strongly enhanced by increasing cell density $[32,33]$.

Here, we use a computational model of CIL, based on the experimental observations described above, to investigate the mechanisms responsible for the emergent collective migration of cells. Exploiting similarities to models of animal migration, we analyse quantitatively the conditions and characteristics of collective migration of cell populations. We investigate the relationship between the statistics of pairwise interactions and the overall group migration, and study the influence of the highly localized tactile interactions on chemotaxis to determine the relative role of individual chemotactic abilities versus cell-cell social interactions. We note, that the aim of this model is not to reproduce the migration of a particular cell type in a specific biological context, but to analyse the role of one particular type of interaction, CIL, in contributing to collective migration while we neglect other type of interactions, e.g. chemo-attraction and cell-cell adhesion.

\section{The model}

We consider a computational model of cell migration in a periodic domain based on a simple tractable interpretation of CIL. In this model cells are composed of a circular core surrounded by a deformable region representing cell protrusions. The overall cell shapes are modelled as closed contours in two dimensions. All cells in our simulations are polarized. The freely moving cell has a front-back asymmetry with protrusions concentrated at the front. We initially define the shape of the cell in polar coordinates with a Gaussian function of the angle $\theta$ as:

$P\left(\theta ; \theta_{0}\right)=R_{0}+A \mathrm{e}^{-\left(\theta-\theta_{0}\right)^{2} / 2 \omega^{2}}, \quad \theta_{0}-\pi<\theta<\theta_{0}+\pi$

where $R_{0}$ is the radius of the core of the cell, $\theta_{0}$ is the instantaneous cell orientation and $\omega$ and $A$ are cell shape parameters. In the numerical simulations the contour is represented by a discrete set of $m$ points corresponding to equally distributed orientations: $\theta_{k}=2 \pi k / m, k=1, \ldots, m$. We use $m=50$ in our simulations. Figures $1(a)-(c)$ shows examples of cell shapes corresponding to different parameter values. The cell shape parameters $\omega$ and $A$ are varied such that the cell area remains constant.

To relate the motion of the cell to its shape, we assume that the protrusions impart a radial force on the cell in the direction of their growth proportionally to their length. Thus we define the velocity of a cell to be proportional with the resultant contributions from protrusions in all directions about the centre of the cell:

$$
\mathbf{v}(t)=v_{0} \int_{\theta_{0}-\pi}^{\theta_{0}+\pi}\left(P(\theta)-R_{0}\right) \mathbf{n}_{\theta} \mathrm{d} \theta
$$

where $\mathbf{n}_{\theta}$ is the radial unit vector in the direction $\theta$ and the prefactor $v_{0}$ is a constant. Note that the cell shape affects cell speed and speed increases as $\omega$ decreases and $A$ increases. In the absence of contact interactions a single cell moves with a 


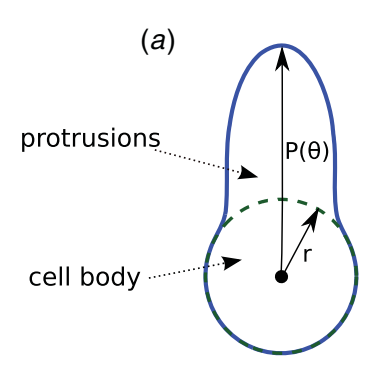

(b)

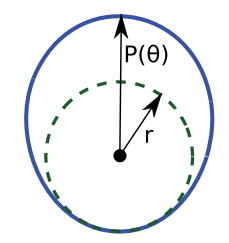

$(d)$
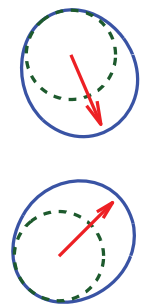

(e)

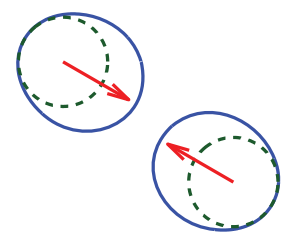

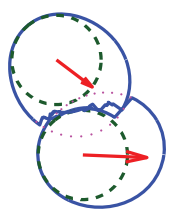

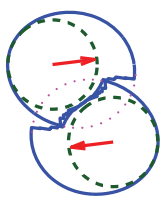

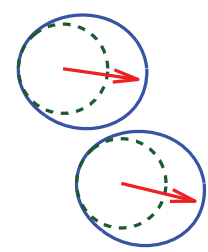

(c)
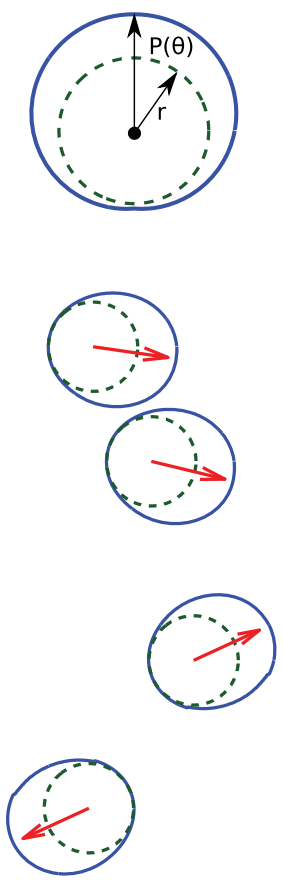

Figure 1. (a)-(c) Varying $\omega$ and $A$ yields different cell shapes (each with $\theta_{0}=\frac{\pi}{2}$ ). (a) $\omega=0.3$ and $A=1.0$. (b) $\omega=0.9$ and $A=0.44$. (c) $\omega=1.5$ and $A=0.3$. (d), (e) Examples of pairwise collisions: when two cells touch a portion of their protrusions overlap. CIL causes the cells to lose protrusions and, as a result, the cells redirect their motion. The cell shape is restored symmetrically about the new angle of motion. The red arrows show the cells' velocity. (d) Glancing collision. (e) Head on collision.

constant velocity and its orientation $\theta_{0}$ changes stochastically according to a Wiener process:

$$
\frac{\mathrm{d} \theta_{0}(t)}{\mathrm{d} t}=\eta(t), \quad\langle\eta(t) \eta(t-\tau)\rangle=\sigma^{2} \delta(\tau),
$$

where $\eta(t)$ is an uncorrelated white noise and $\sigma$ is the noise intensity. A target Gaussian shape function $P_{0}\left(\theta ; \theta_{0}(t)\right)$ is then defined with the actual orientation using (1) and the cell shape relaxes towards this new Gaussian contour according to:

$$
\frac{\partial P(\theta, t)}{\partial t}=-\gamma\left[P(\theta, t)-P_{0}\left(\theta ; \theta_{0}(t)\right)\right]
$$

where $\gamma$ determines the rate of regrowth and $\theta_{0}(t)$ is the instantaneous direction of motion at time $t$.

When two cells meet the protrusions are withdrawn within the area of contact, resulting in a reorientation of the direction of motion. In the computational implementation, overlapping segments of the cells are detected and in the corresponding radial directions the contours are reset to the core radius. The collapse of protrusions breaks the symmetry of the cell with respect to the original orientation axis and changes the direction of motion. The loss of protrusions during cell contact typically leads to a temporary decrease in the speed of locomotion. Following the reorientation due to the modified shape of the contour the cells move away from each other and gradually regain their normal shape and speed by reforming protrusions symmetrically along the new direction of movement (figures $1(d)$ and $(e)$ ). In the numerical simulations the velocity vectors and cell contours are updated using discrete time steps. At each step first the velocity vector is determined from the newly updated cell shape $P(\theta)$ according to (2), and the orientation of the velocity vector is combined additively with the noise corresponding to (3). The resulting direction $\theta_{0}(t)$ determines the orientation of the target Gaussian cell shape $P_{0}\left(\theta_{0}(t)\right)$ in the relaxation dynamics (4).

We note that real cell protrusions are typically more irregular and dynamically changing [15, 16]. Recent work by Vedel et al [34] presents a similar computational model of cells migrating that interact via CIL. In their model multiple short-lived protrusions form in spikes about the cell body. The contour defined by (1) can be considered as a time-averaged representation of the distribution of such random extensions around the cell.

\section{Results}

\subsection{Collective migration}

Using our cell interaction model we performed simulations of groups of interacting cells. To avoid dispersion and maintain a constant density, we assume that the cells are restricted to a finite domain. The results of simulations of 200 cells in a periodic domain are presented in figure 2 at four different densities (see supplementary material, movies S1 and S2 (available from stacks.iop.org/PhysBio/10/046002/mmedia)). The density is calculated as the number of cells per unit area of the domain. First we carried out simulations in the absence of noise $(\sigma=0)$. As in previous models of flocking behaviour 

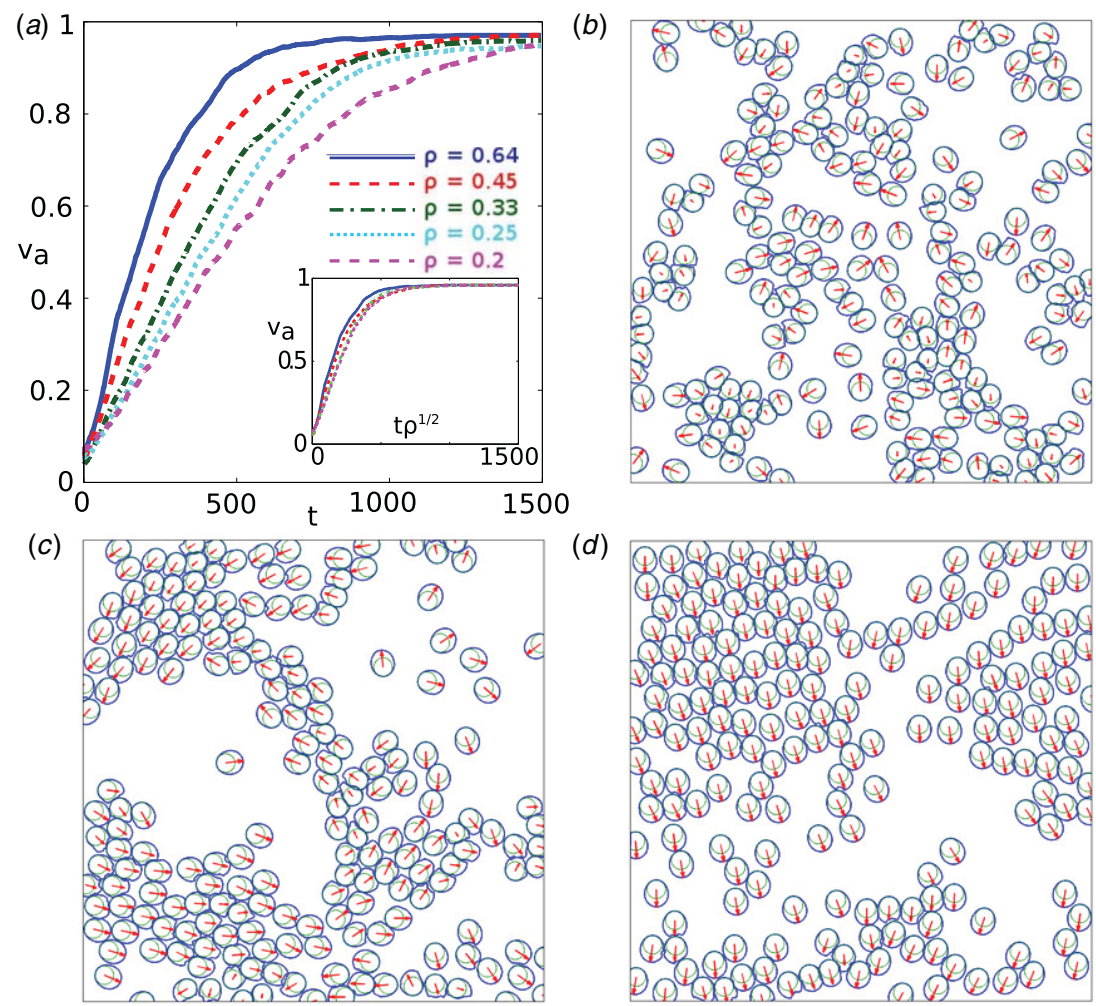

Figure 2. (a) The order parameter, $v_{a}$ versus time for 200 cells at different densities with noise, $\sigma=0$. In each simulation the cells align in a random direction. The transient time varies as the density is changed. $(b)-(d)$ Snapshots of 200 cells at $\rho=0.33$ and $\sigma=0$ with random initial positions and orientations. Inset: the order parameter versus time adjusted to the mean time between collisions. The curves approximately overlap. (b) $t=50,(c) t=500,(d) t=1500$. The red arrows indicate the instantaneous velocity of each cell. $A=0.43$, $\omega=0.9, R_{0}=0.5, \gamma=0.1$ and $v_{0}=1$.

$[9,10]$ we characterize the collective alignment of the cell population by the order parameter $v_{a}$ defined as:

$$
v_{a}=\frac{\left|\sum_{i=1}^{n} \mathbf{v}_{i}\right|}{\sum_{i=1}^{n}\left|\mathbf{v}_{i}\right|}
$$

where $\mathbf{v}_{i}$ is the velocity of the $i$ th cell. The order parameter varies between 0 and 1 and measures the degree of collective alignment of the cells. Initially cells are given random position and orientation.

As shown in figure 2 in the absence of noise the cells gradually form a perfectly aligned group moving in a randomly chosen common direction. Although asymptotically the order parameter tends to unity in all simulations, the transient time necessary to reach the ordered state increases as the cell density is reduced. This is expected since at lower densities the collisions are less frequent, thus the consensus direction is reached over a longer time since the local orientations propagate more slowly through the group.

The ordered collective motion also develops in a circular domain (see supplementary material, movie S3 (available from stacks.iop.org/PhysBio/10/046002/mmedia)) where the perimeter of the region is made up of immotile cells that the moving cells interact with via CIL. In this case the collective alignment is described by the group angular momentum defined by:

$$
v_{c}=\frac{1}{n}\left|\sum_{i=1}^{n} \sin \left(\beta_{i}\right)\right|
$$

where $\beta_{i}$ is the angle between the direction of motion of the $i$ th cell and the radial direction relative to the centre of the circular domain. The tendency for motile cells in confinement to circulate collectively as a group has been described in [35].

\subsection{Phase transition and shape dependence}

Previous work on flocking models with non-local interactions have shown that varying the strength of the stochasticity of self-propelled point-like particles leads to a transition between organized collective motion and random orientations [9, 35]. This transition has similarities to the phase transition in ferromagnets when the temperature passes through the critical point. To test the sensitivity of organized collective movement in our model of cell migration to the strength of stochastic effects we carried out a set of simulations with increasing noise levels. Figure 3(a) presents the order parameter at stationary state as a function of noise amplitude, $\sigma$, for four different cell shapes. These results clearly indicate a transition similar to the self-propelled particle models, i.e. there is a sharp drop in collective alignment of cells as the noise level approaches a critical value above which the group can no longer align. Note, that the noise intensity at which the transition takes place depends on the cell shape parameters, suggesting that certain cell shapes (i.e. protrusion distributions) have better ability for generating robust global alignment, than do others. We find that broadly distributed protrusions, corresponding to larger values of $\omega$ (as in figures $1(b)$ and $(c)$ ), in general, produce 

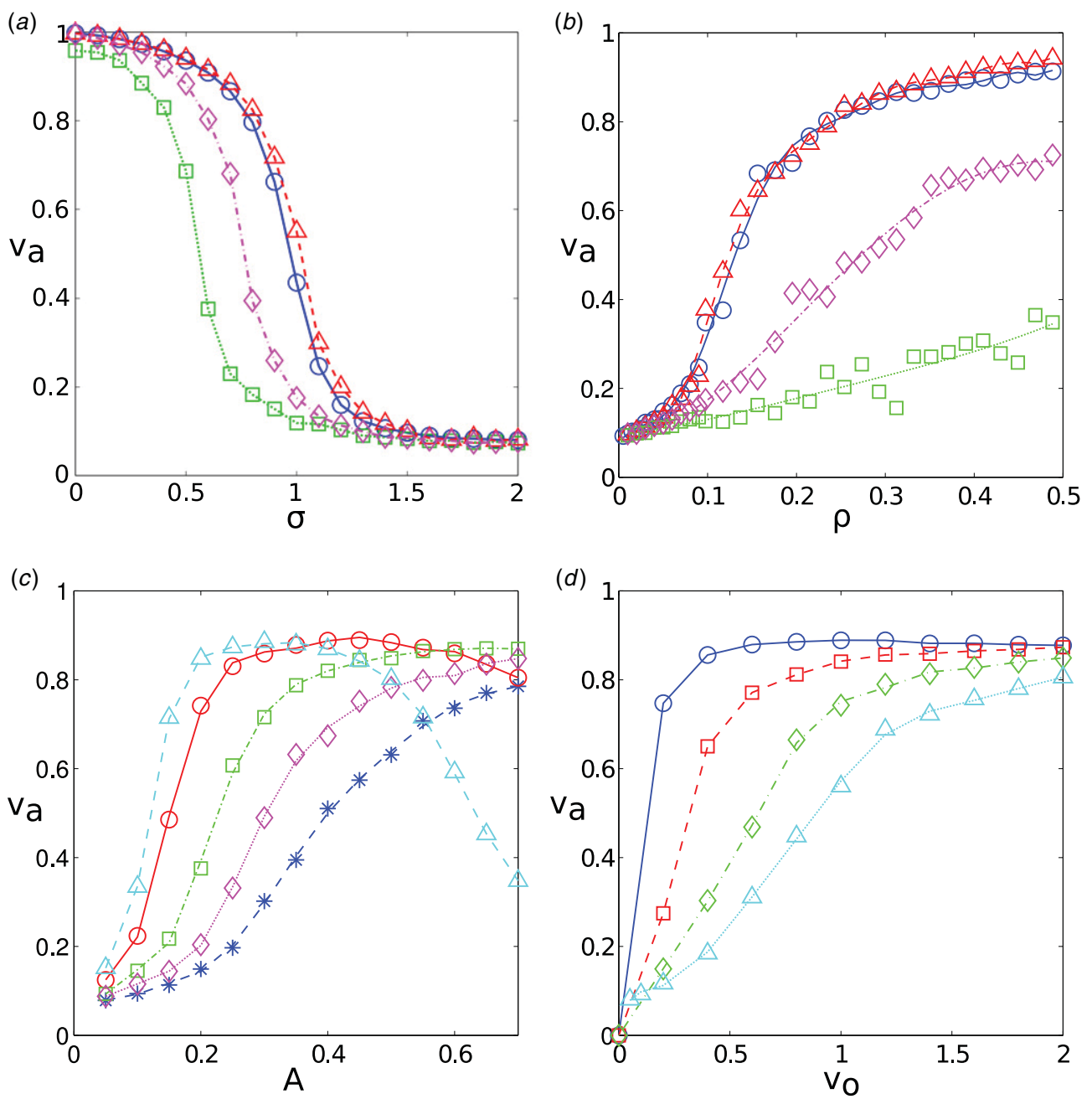

Figure 3. (a) The order parameter, $v_{a}$ in the stationary state versus noise intensity $\sigma$ for four different cell shapes in periodic domain with $\rho=0.32$. As the noise is increased there is a sharp transition from collective alignment to random movement. $(b)$ The order parameter $v_{a}$ in the stationary state versus density $\rho$ where $\sigma=0.7$. Alignment improves as density is increased. $\triangle: \omega=1.5$ and $A=0.32$ (red), O: $\omega=0.9$ and $A=0.44$ (blue), $\diamond: \omega=0.5$ and $A=0.56$ (magenta), $\square: \omega=0.3$ and $A=0.74$ (green). (c) $v_{a}$ versus $A$ protrusion length, $\omega=0.9, \sigma=0.8$. $\triangle$ (cyan): $\rho=0.8$; O (red): $\rho=0.64 ; \square$ (green): $\rho=0.45 ; \diamond$ (magenta): $\rho=0.33$; (blue): $\rho=0.25$. (d) $v_{a}$ versus $v_{o}$ cell speed, $A=0.44, \omega=0.9, \sigma=0.8$. O (blue): $\rho=0.64 ; \square$ (red): $\rho=0.45 ; \diamond$ (green): $\rho=0.33 ; \triangle$ (cyan): $\rho=0.25$.

better alignment for a given level of noise, than does having strongly polarized cell shapes with protrusions concentrated at the front of the cell even though such cell shapes yield faster moving cells (figure $1(a)$ ). This appears to be a consequence of the need to balance cell polarization and directional motility with the need for social cell-cell interactions as sensed by lateral protrusions, in order to maintain the collective direction in the presence of noise.

A similar, but more gradual, transition also takes place when the cell density is varied at a fixed value of $\sigma$, i.e. higher density corresponds to stronger alignment (see figure $3(b)$ ). This is again in agreement with particle based flocking models, as well as experimental data on swarming locusts [36], suggesting that this cell model belongs to the same universality class, in spite of the absence of non-local interactions. To further investigate the shape dependence of the collective motion figure 3(c) show the order parameter as a function of maximum protrusion length $A$ while the width $\omega$ is kept constant at different cell densities. The results show that although a stronger polarization enhances global alignment any polarized cells form an ordered state when the density is sufficiently high. Note at high density and high protrusion length $v_{a}$ drops as there is no longer the free space for protrusions to grow into and CIL prevents the cells from moving. Figure 3(d) shows the order parameter as a function of the cell speed parameter illustrating that a stronger alignment is reached when the cells move faster, i.e. when the frequency of cell collisions increases.

Recent results based on a self-propelled particle model with only pairwise short-range interaction [37] suggest that collective alignment can arise in general from inelastic collisions, i.e. when the relative angle between the orientation of two particles is reduced as a result of their interaction. Since the cells in our model are represented by spatially extended contours, in this case there is no unique relationship between the orientations of two cells before and after their interactions. However, we can characterize the cell-cell interaction by averaging over the possible pairwise collisions.

To investigate how the collective alignment depends on the cell shape we carried out simulations of pairwise collisions 

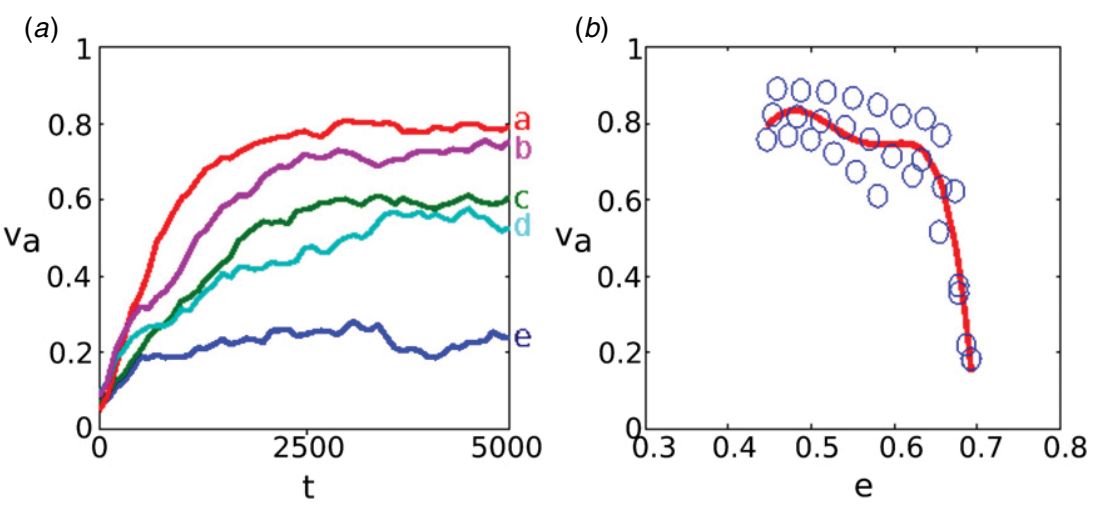

Figure 4. (a) The order parameter of groups of 200 cells with shape parameters shown in table 1 . In the presence of noise, $\sigma=0.7$ and density, $\rho=0.32$. The shapes corresponding to lower values of $e$ produce stronger alignment. (b) Stationary state order parameter versus $e$ for many different cell shapes in the range $A=0.3-0.9, \omega=0.3-1.65$ with noise, $\sigma=0.7$.

Table 1. Restitution coefficient of pairwise collisions, $e$ and stationary state order parameter, $v_{a}$ (where $n=200, \rho=0.32$ and $\sigma=0.7$ ) measured for different cell shapes (see figure 4).

\begin{tabular}{ccccc}
\hline & $\omega$ & $A$ & $e$ & $v_{a}$ \\
\hline (a) & 1.5 & 0.3 & 0.47 & 0.77 \\
(b) & 1.2 & 0.3 & 0.52 & 0.72 \\
(c) & 0.9 & 0.3 & 0.58 & 0.61 \\
(d) & 0.6 & 0.5 & 0.65 & 0.52 \\
(e) & 0.3 & 0.7 & 0.69 & 0.18 \\
\hline
\end{tabular}

in which the relative angle between the orientation of two cells before collision, $\Delta \theta_{i}$, was varied from 0 to $\pi$ in small increments so that the collisions are initially glancing and finally head on. At each choice of initial relative angle a set of collisions are carried out varying the initial starting position so that the point of contact ranges from the rear to the front of the cells. We measured the final relative angles, $\Delta \theta_{f}$, and calculated its average value over the number of collisions carried out at each $\Delta \theta_{i}$, to obtain the average final relative angle after collision: $\left\langle\Delta \theta_{f} \mid \Delta \theta_{i}\right\rangle=f\left(\Delta \theta_{i}\right)$. We define the restitution coefficient of cell collisions as the ratio between the average final and initial angles:

$$
e=\frac{1}{M} \sum_{j=1}^{M} \frac{\left\langle\Delta \theta_{f} \mid \Delta \theta_{i}=\pi j / M\right\rangle}{\pi j / M} .
$$

This quantity provides a statistical measure of the average tendency to reduce the relative angle between two cells in pairwise collisions. The values obtained for different cell shapes are shown in table 1 . Smaller values correspond to stronger average pairwise alignment that are expected to increase the velocity correlations in a cell population. This is confirmed in figure 4 where we show the order parameter of groups of cells in the presence of noise for the same cell shapes. Comparing the values of $e$ and the corresponding order parameters in the stationary state shows that cell shapes that produce stronger average alignment in pairwise collisions also create more coherent collective migration in a cell population.

\subsection{Collective chemotaxis}

In this section we investigate the role of the previously described collective motion in enhancing the overall chemotactic response of the cell population, a property that has been observed in recent experimental studies [32, 33]. Since here we focus on the collective behaviour we do not represent explicitly the processes that generate the chemotactic response at the level of individual cells. (For a recent model that describes the effect of chemoattractant on cell protrusions and motility in single cells see [17].) Instead we model the overall chemotactic response of cells by assuming that they turn towards the direction of a fixed gradient, so that in between collisions the orientation of a freely moving cell follows the equation:

$$
\frac{\mathrm{d} \theta_{0}}{\mathrm{~d} t}=-\alpha \sin \left(\theta_{0}-\theta_{c}\right)+\eta(t)
$$

where $\alpha$ is the maximum turning rate due to chemotaxis, $\theta_{c}$ is the direction of the gradient and $\eta(t)$ is a white noise (as in equation (1) above) representing the combined effects of stochastic cell movement and errors from detecting the gradient.

Following [12, 13], we characterize the ability of cells to navigate in the direction of the gradient by the chemotactic efficiency, $\lambda$, calculated as the normalized projection of their displacement in the direction $\theta_{c}$, defined as:

$$
\lambda=\left\langle\cos \left(\theta_{0}(t)-\theta_{c}\right)\right\rangle
$$

where $\langle\ldots\rangle$ represents a time average. The maximum value of the chemotactic efficiency is $\lambda=1$ when the cell is aligned perfectly in the direction of the gradient. For single cells $\lambda$ increases with the turning rate $\alpha$ and decreases when the noise intensity is increased. The chemotactic efficiency of cell groups is calculated by averaging the efficiency of individual cells: $\Gamma=\sum_{i=1}^{n} \lambda_{i} / n$, where $n$ is the number of cells and $\lambda_{i}$ is the chemotactic efficiency of the $i$ th cell calculated from (9) above.

Simulations were performed for various cell densities and for different values of the chemotactic turning rate $\alpha$. Results of the simulations (figure 5(a)) show that in all cases after a certain transient time the chemotactic efficiency of the group reaches approximately the same value as the order parameter of the same cell population without an external gradient. Thus the chemotactic efficiency of the group is determined by the ability of cells to coordinate their direction of movement, that depends on density and cell shape, but it is essentially 

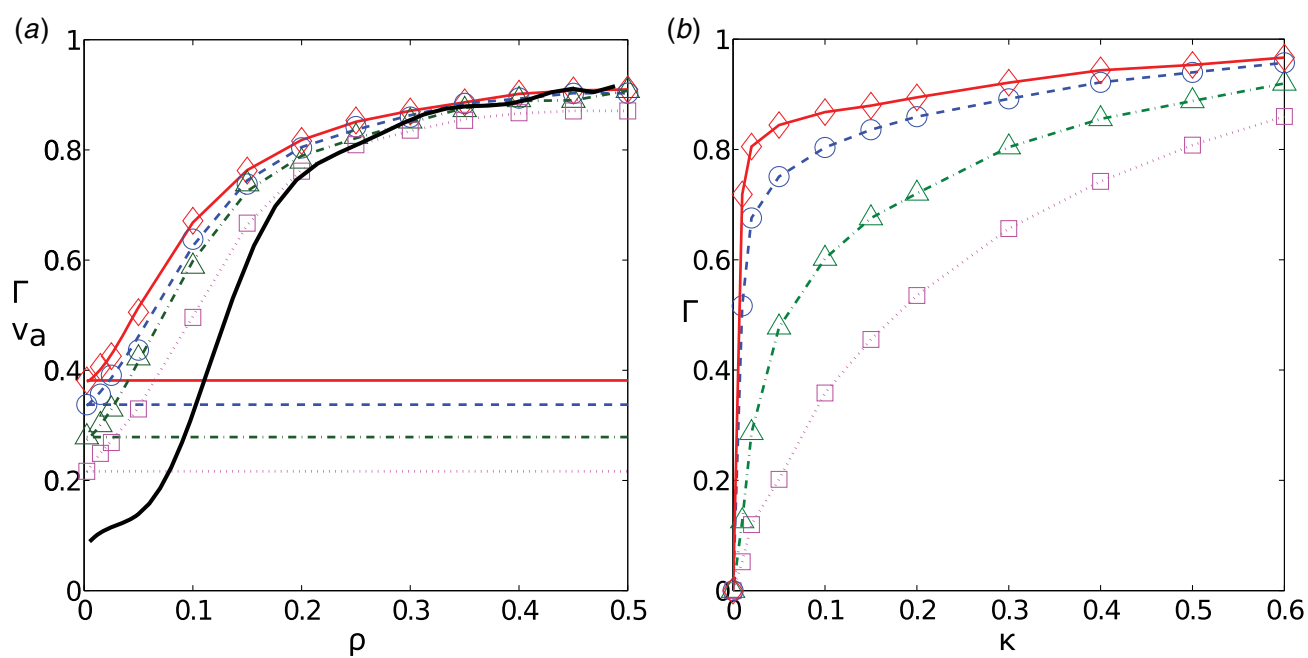

Figure 5. (a) Chemotactic efficiency, $\Gamma$ versus density of populations of 200 cells migrating toward a chemo-attractant with different turning rates $\alpha=0.012$ (magenta $\square$ ), 0.015 (green $\triangle$ ), 0.018 (blue $\mathrm{O}$ ) and 0.021 (red $\diamond$ ) with $\sigma=0.7$ compared with the order parameter in the absence of chemo-attractant (black solid line). The horizontal lines show the chemotactic efficiency of single cells for comparison, for the same values of $\alpha$ and $\sigma$. (b) Chemotactic efficiency of 100 cells versus proportion of leaders, $\kappa$ at density, $\rho=0.05$ (magenta $\square$ ), 0.1 (green $\triangle$ ), 0.2 (blue $\mathrm{O}$ ) and 0.3 (red $\diamond$ ) with $\sigma=0.7$. Note that a very small proportion of leader cells, that maintain their orientation along the gradient, can produce near maximum chemotactic efficiency in the group (see supplementary movie S4 (available from stacks.iop.org/PhysBio/10/046002/mmedia)).

independent of the ability of cells to turn in the direction of the gradient. This is because, non-chemotactic cells align themselves spontaneously in an arbitrary direction, and any small bias acting at the level of individual cells is sufficient to turn such ordered group into the direction of the gradient. The only consequence of the turning rate $\alpha$ is that the time needed to reach the direction of the gradient is longer when the group is composed of cells with weaker chemotactic response.

Figure 5(a) shows that the collective migration leads to a strong enhancement of the chemotactic efficiency of a group with respect to single cells, in agreement with the experimental observations [32]. Note, that the same type of collective chemotaxis also appears in heterogeneous cell populations, where only a very small proportion of 'leader cells', that have the ability to sense the chemical gradient, are sufficient to steer the group in the direction of the chemoattractant (see figure 5(b)), and supplementary movie S4 (available from stacks.iop.org/PhysBio/10/046002/mmedia)). Thus the underlying newly observed phenomena of collective chemotaxis in cell populations is in fact closely related to the one described earlier for migrating animal groups guided by a small proportion of leaders [11-13].

\section{Discussion}

Many biological systems consist of individuals interacting with one another and responding to the conditions of their environment. Often these processes are interrelated and it is the collective behaviour of the system that determines the effectiveness of the response. This form of emergent response to environmental information is most commonly studied in whole organisms, for example schools of fish that respond to the approach of a predator [38] or migratory species that track a resource gradient. However, it is increasingly becoming evident that underlying principles exist that extend across multiple scales and taxa, from interacting animal populations to the functioning of cells.

In this work we have introduced a simple, yet realistic model of cellular interaction, inspired by experimental work on the phenomena of CIL. Our model represents the behaviour of individual cells from a mechanistic perspective; collisions induce a reflexive reshaping of cell protrusions and a consequent change in orientation. We have shown that depending on the cell shape such interaction may lead to inelastic collisions that produce increased alignment. Thus the contact based cell interaction is analogous with the alignment rule of the particle based flocking models $[9,10]$. Consequently, at the collective level, pairwise interactions lead to global cohesion, hence CIL leads to behaviour that is qualitatively equivalent to that observed in classic models of animal grouping, despite interaction ranges being restricted to the extent of individual cells. For cell populations interacting via CIL, as in larger organisms, there is a phase transition from disorder to an ordered regime when local noise and overall density is varied. An example of such transition can be seen in neural crest cells where the chemokine Sdf1 increases the activity of small GTPases and stabilises cell protrusions [32], effectively reducing the noise of the individual cell motility that induces coherent collective migration.

Our model also provides support for the use of explicit alignment rule in particle based models of neural crest cell migration [26]. Recent studies have found that co-attraction between cells may play a role in collective migration [26, 27]. This is also consistent with the framework of flocking models $[9,10]$. While in the model that we considered here the density of cells was maintained by closed boundaries; in an unconstrained system attractive interactions are necessary to 
avoid dispersion. The strength of such chemo-attraction then regulates the density of cells and therefore determines the characteristics of collective migration.

When cell functioning is dependent on chemotaxis the possibility of spontaneous self-organization has important implications. In situations when cell density is high and environmental sensing is imperfect, it is aggregate quantities that determine an individual cells ability to accurately respond to chemical gradients. For homogeneous populations, after relaxation of transients, the accuracy achieved by a single cell is equivalent to the degree of collective cohesion. This quantity is affected by cell shape (through its influence on pairwise interactions) and overall cell density, but notably, not by the detection capacity of individual cells. The accuracy of chemotaxis is therefore determined by the collective system and not by the properties of individuals in isolation.

\section{Acknowledgments}

We would like to thank V Lobaskin and R Mayor for useful comments and discussions.

\section{References}

[1] Montell D J 2008 Morphogenetic cell movements: diversity from modular mechanical properties Science 322 1502-5

[2] Rørth P 2009 Annu. Rev. Cell Dev. Biol. 25 407-29

[3] Friedl P and Gilmour D 2009 Nature Rev. Mol. Cell Biol. 10 445-57

[4] Méhes E, Mones E, Németh V and Vicsek T 2012 PLoS ONE 7 e31711

[5] Kulesa P M and Fraser S E 1998 Dev. Biol. 204 327-44

[6] Teddy J M and Kulesa P M 2004 Development 131 6141-51

[7] Friedl P and Wolf K 2003 Nature Rev. Cancer 3 362-74

[8] Deisboeck T S and Couzin I D 2009 BioEssays 31 190-7

[9] Vicsek T, Czirok A, Ben-Jacob E, Cohen I and Shochet O 1995 Phys. Rev. Lett. 75 1226-9

[10] Couzin I D, Krause J, James R, Ruxton G D and Franks N R 2002 J. Theor. Biol. $2181-11$

[11] Couzin I D, Krause J, Franks N R and Levin A S 2005 Nature 433 513-6

[12] Guttal V and Couzin I D 2010 Proc. Natl Acad. Sci. $10716172-7$
[13] Torney C, Levin S A and Couzin I D 2010 Proc. Natl Acad. Sci. 107 20394-9

[14] Ananthakrishnan R and Ehrlicher A 2007 Int. J. Biol. Sci. 3 303-17

[15] Bosgraaf L and van Haastert P J M 2009 PLoS ONE 4 e5253

[16] Insall R H 2010 Nature Rev. Mol. Cell Biol. 11 453-8

[17] Neilson M P, Veltman D M, van Haastert P J M, Webb S D, Mackenzie J A and Insall R H 2011 PLoS Biol. 9 e1000618

[18] Trepat X, Wasserman M R, Angelini T E, Millet E, Weitz D A, Butler J P and Fredberg J 2009 Nature Phys. 5 426-30

[19] Bindschadler M and McGrath J 2007 J. Cell Sci. 120 876-84

[20] Farhadifar R, Röper J-C, Aigouy B, Eaton S and Jülicher F 2007 Curr. Biol. 17 2095-210

[21] Puliafito A, Hufnagel L, Neveu P, Streichan S, Sigal A, Fygenson D K and Shraiman B I 2012 Collective and single cell behavior in epithelial contact inhibition Proc. Natl Acad. Sci. 109 739-44

[22] Graner F and Glazier J A 1992 Phys. Rev. Lett. 69 2013-6

[23] Szabo A, Unnep R, Mehes E, Twal W O, Argraves W S, Cao Y and Czirok A 2010 Collective cell motion in endothelial monolayers Phys. Biol. 7046007

[24] Kabla A J 2012 J.R. Soc. Interface 9 3268-78

[25] De Calisto J, Araya C, Marchant L, Riaz C F and Mayor R 2005 Development 132 2587-97

[26] Carmona-Fontaine C, Theveneau E, Tzekou A, Tada A, Woods M and Page K 2011 Dev. Cell 21 1026-37

[27] McLennan R, Dyson L, Prather K, Morrison J, Baker R and Maini P 2012 Development 139 2935-44

[28] Abercrombie M and Heaysman J E M 1953 Exp. Cell Res. $5111-31$

[29] Abercrombie M 1979 Nature 281 259-62

[30] Carmona-Fontaine C, Matthews H K, Kuriyama S, Moreno M, Dunn G A, Parsons M, Stern C D and Mayor R 2008 Nature $456957-61$

[31] Mayor R and Carmona-Fontaine C 2010 Trends Cell Biol. $20319-28$

[32] Theveneau E, Marchant L, Kuriyama S, Gull M, Moepps B, Parsons M and Mayor R 2010 Dev. Cell 19 39-53

[33] Theveneau E and Mayor R 2010 Small GTPases 1 113-7

[34] Vedel S, Tay S, Johnston D M, Bruus H and Quake S R 2012 Proc. Natl Acad. Sci. 110 129-34

[35] Szabó B, Szöllösi G J, Gönci B, Jurányi Z, Selmeczi D and Vicsek T 2006 Phys. Rev. E 74061908

[36] Buhl J, Sumpter D J T, Couzin I D, Hale J J, Despland E, Miller E R and Simpson S J 2006 Science 3125778

[37] Grossman D, Aronson I S and Ben-Jacob E 2008 New J. Phys. 10023036

[38] Handegard N O, Boswell K M, Ioannou C C, Leblanc S P, Tjostheim D B and Couzin I D 2012 Curr. Biol. 22 1213-7 